\title{
Characterizing stress tolerance: "controllability awareness" and its relationship to perceived stress and reported health
}

\author{
Josephine Todrank Heth ${ }^{\mathrm{a}, *}$, Eli Somer ${ }^{\mathrm{b}}$ \\ ${ }^{a}$ Ray D. Wolfe Center for the Study of Psychology Stress, University of Haifa, Haifa 31905, Israel \\ ${ }^{\mathrm{b}}$ School of Social Work, University of Haifa, Haifa 31905, Israel
}

Received 30 March 2001; received in revised form 14 October 2001; accepted 12 November 2001

\begin{abstract}
"Controllability awareness" describes the extent to which an individual's responses to life situations reflect attention to distinctions between controllable and uncontrollable aspects of potential outcomes. The construct recognizes four aspects of controllability: personal control of outcomes, shared control of outcomes, others in responsibility, and uncontrollable/unpredictable outcomes. Lacking a suitable measurement instrument, the Controllability Awareness Inventory (CAI) was developed and used to assess controllability awareness as a characteristic of stress tolerance. Results indicate that individuals with a greater tendency to respond based on an awareness of the controllability of potential outcomes perceive their lives as less stressful, report themselves to be in better health, and think in ways that enable them to manage environmental demands more effectively. Controllability awareness training to help reduce perceived stress and promote health is discussed. (C) 2002 Elsevier Science Ltd. All rights reserved.
\end{abstract}

Keywords: Controllability; Stress tolerance; Perceived stress; Health; Coping

\section{Introduction}

Some individuals appraise life situations as challenges while others see the same types of situations as threatening. That some individuals tolerate potential stressors more effectively than others do has been sufficiently intriguing to inspire substantial theoretical and practical investigations, particularly because of the clear connection between psychological stress and illness (e.g. Cohen \& Herbert, 1996). Despite the great contributions of "hardiness" (Kobasa, 1979), "sense of coherence" (Antonovsky, 1987), "self-efficacy" (Bandura, 1982), "optimistic explanatory style" (Peterson \& Seligman, 1987), "personal control” (Peterson \& Stunkard, 1989;

\footnotetext{
* Corresponding author.

E-mail address: heth@research.haifa.ac.il (J. Todrank Heth).
} 
Thompson \& Spacapan, 1991), and “internal locus of control” (Levenson, 1981; Rotter, 1966), there is still room for clarifying individual differences in stress tolerance and their relationship to physical and mental health. We are interested in exploring whether those who distinguish between controllable and uncontrollable aspects of situations and act or accept accordingly are more stress tolerant than those who do not make such distinctions. In other words, does "controllability awareness" contribute to appraising situations as challenging rather than threatening? The studies reported here are part of a continuing research effort to identify characteristics of stress tolerance, focusing particularly on the importance of the awareness of the extent to which potential outcomes are or are not controllable.

\subsection{Coping strategies and coping styles}

Previous research has identified different coping strategies and investigated how certain coping styles are associated with personality characteristics. The most enduring model of coping, developed by Lazarus and his colleagues (Lazarus \& Folkman, 1984), sees the individual in dynamic interaction with an ever-changing environment that can be both challenging and threatening, and divides coping responses into two broad categories: problem-focused coping involves direct action to solve the problem or change the outcome of the situation; emotion-focused coping involves strategies to deal with the emotional impact of the problem or situation. When the individual's coping resources are perceived as sufficient to meet the environmental demands, the situation is appraised as challenging, whereas insufficient coping resources lead to threat appraisals (Lazarus, 1991). Because appropriate coping strategies depend on the particular environmental demands, coping responses are assessed in specific situations when using this approach (Folkman, 1984). Another approach involves assessing how different personality types respond to environmental demands, the contention being that coping styles or dispositions are more predictive of coping responses than coping strategies. These researchers focus their investigations on distinctions such as those between "monitors" (who focus in on the details of the situation) and "blunters" (who distance themselves from the impact of the situation; Miller, 1992), between "internals" (who attribute the causality of outcomes mostly to themselves) and "externals" (who attribute causality of outcomes to others or to chance; Levenson, 1981; Rotter, 1966), between optimists and pessimists (Carver, Scheier, \& Weintraub, 1989; Scheier, Weintraub, \& Carver, 1986), or between varying degrees of self-efficacy (a person's belief in his/her ability to achieve a desired outcome; Bandura, 1982).

In all likelihood, the individual chooses coping strategies from his/her set of coping resources depending on the appraisals of the specific situation while the resources available in the individual's particular skill set and the probability of selecting any particular strategy depend on personality characteristics. There is evidence from the coping literature that is consistent with this possibility. For example, optimists tend to use problem-focused coping strategies when the situation is controllable and acceptance (an emotion-focused coping strategy) when the situation appears uncontrollable (Carver \& Scheier, 1994; Scheier \& Carver, 1992). The concepts of "constructive thinking" (the ability to solve everyday problems while minimizing experienced stress; Epstein \& Katz, 1992; Epstein \& Meier, 1989) and "adaptiveness" (coping consistently to reduce or not aggravate distress; Kohn, 1996) recognize, or at least imply, that the most effective "style" involves using multiple situation-dependent strategies. Thus, coping responses are probably a combination of styles and strategies. 


\subsection{A new look at controllability}

Although it is possible to determine objectively whether potential outcomes can or cannot be influenced by human actions, most life situations are complicated combinations of aspects of sufficient social complexity that it may be difficult to determine the relative influence of the various individuals involved and the likelihood of success. Extending the locus of control concept from a distinction between internal and external causality (Rotter, 1966) to distinctions among internality, powerful others, and chance (Levenson, 1981) was a positive step in understanding the importance of controllability in human behavior. Although assessing the tendency to make causality attributions as internal or external in ambiguous situations is instructive, it may be equally instructive to investigate the tendency to sort out the controllable and uncontrollable aspects of less ambiguous but complicated social situations. Often in human society, people do not have full personal control over a potential outcome, but rather must work together to achieve a desired outcome, each one being in control of his/her part. Controllability may become more useful in explaining human behavior after incorporating the richness of social relationships into the construct through including the concept of shared control. People usually are not at the mercy of "powerful others", but frequently experience situations where others are primarily responsible for the outcome. Taking this social complexity into account, intended outcomes may be classified more accurately as having four potential controllability aspects: those that are under one's personal control; those that are in others' control; those in which the control is shared by oneself and others; those that are not predictable or controllable.

\subsection{Controllability awareness and stress tolerance}

Because the extent to which individuals can accurately determine their personal control over events affects the efficiency with which they can use their energy to produce the desired outcomes (Skinner, 1996), it follows that individuals who are aware of the controllability of intended outcomes and act based on their controllability analysis would manage environmental demands more effectively. By being aware of the controllability of the potential outcomes, individuals should be more likely to appraise life situations as challenging rather than threatening, and therefore they should experience less stress. By perceiving their life situations as more manageable because they are aware of what they can and cannot achieve, controllability aware individuals should be able to minimize the deleterious effects of stress responses that accompany threat appraisals (Lazarus, 1991). The hypothesis motivating this investigation is that individuals who deal with life situations by focusing their energy on acting in response to the aspects of situations that they can control or influence rather than wasting energy on the aspects that cannot be controlled should enhance their chances of feeling healthy compared with individuals who do not use controllability awareness. Individuals who are stress-tolerant because of controllability awareness should be characterized by three distinguishing features: (1) the ability to respond based on distinctions between the controllable and uncontrollable aspects of life situations even when those situations are complex; (2) the ability to handle life's challenges by identifying practical situationspecific solutions that can be implemented efficiently, either alone or in cooperation with others; and, (3) the ability to accept those things that it would be futile to attempt to change, either because they are uncontrollable or because they are someone else's responsibility. In addition, 
being able to use effective action and acceptance simultaneously should further enhance stress tolerance.

Of course, certain aspects of other previously demonstrated health-promoting characteristics should also be evident in stress-tolerant individuals. The purpose of investigating controllability awareness as a distinguishing feature of stress-tolerant individuals is not to diminish the importance of other health-promoting characteristics, but rather to identify a crucial characteristic that may be more amenable to change through training than the previously reported features. Whereas personality and temperament are notoriously difficult to change, attitudes are more tractable, particularly when they are connected with a skill that can be acquired and when accompanied by personal motivation. Using controllability awareness principles is a trainable skill, albeit a more difficult skill for some personality types to acquire than others. Before undertaking the development of a controllability awareness training program, it is important to determine whether controllability awareness is predictably related to perceived stress and health.

Although there are a number of research tools that approximate assessment of the ideas explored here in that they fall under the rubric of "control" or "perceived control" and many that assess different aspects of coping, none assess differential controllability of different aspects within the same situation nor do any address all the aspects of controllability included in the controllability awareness construct. To assess the extent to which individuals' responses to situations reflect a realistic analysis of the controllability of the various potential outcomes, we developed the Controllability Awareness Inventory and then used it to determine whether those who pay attention to controllability and act accordingly are better at meeting environmental demands than those who do not pay attention to controllability.

\section{Methods}

"Controllability Awareness" is an approach to life that should be reflected in relatively more challenge appraisals than threat appraisals irrespective of the severity of the environmental demands, thus it should be possible to assess controllability awareness as effectively in terms of responses to situations encountered on a daily basis as in terms of responses to particularly demanding unusual events. The Controllability Awareness Inventory (CAI) was designed with this in mind. In this study, the CAI was validated, and the results were correlated with results from the Constructive Thinking Inventory (CTI; Epstein \& Meier, 1989), the Personal Functioning Inventory (PFI; Kohn, O’Brien-Wood, Pickering, \& DeCiceo, 1998), the Internal, Powerful Others, and Chance scales (IPC; Levenson, 1981), the hardiness scale (Kobasa, 1979), the Perceived Stress Scale (PSS; Cohen, Kamarck, \& Mermelstein, 1983), and the Cohen-Hoberman Inventory of Physical Symptoms (CHIPS; Cohen \& Hoberman, 1983).

\subsection{Participants and procedure}

Forty-seven paid volunteers (24 women and 23 men ranging in age from 18 to 30 with an average age of 22), recruited from the student population of a university in Israel by advertisements on bulletin boards, participated in the study; 25 (12 women and 13 men) returned to complete the retest. Because some of the subjects were Russian immigrants to Israel who may not 
yet have acquired full comprehension of written Hebrew, the validity scale from the CTI, which measures failures of comprehension, was used to screen subjects' responses. The results from five subjects were not included in the analysis because of unacceptably low validity scores. An additional total of 220 students ( 97 women and 38 men, plus five who did not indicate their sex, in their early 20s who were undergraduate students at a university in the United States, and 48 women and 31 men, plus one who did not indicate his/her sex, with an average age of 26 who were undergraduate and graduate students at a university in the New Zealand) completed questionnaires during classes in Psychology.

The first test battery consisted of the Controllability Awareness Inventory (CAI), the 23 items assessing global constructive thinking from the CTI, the PFI, the PSS, and the CHIPS. To avoid possible order effects, subjects completed the CAI first because it was new and had not yet been validated in other studies. Subjects completed the questionnaires (either in English or in Hebrew) in the laboratory. Some subjects returned to the laboratory a week later to complete the CAI retest; these subjects also completed the IPC and the hardiness scale during their second session. A different translator verified the Hebrew translations of all the scales through a back translation into English. Later after the CAI had been validated, students were invited to participate in the second part of the study, and those who agreed were given time to complete the CAI, PSS, and CHIPS during a regularly scheduled class. Unfortunately, it was not possible to assess other subject characteristics in these groups that may have shed light on cultural differences between the samples because of time limitations of the volunteers.

\subsection{Measures}

The concepts of "constructive thinking” (Epstein \& Katz, 1992; Epstein \& Meier, 1989) and "adaptiveness" (Kohn, 1996) led to the development of two measurement tools that are grounded in ideas that are akin to controllability awareness, the Constructive Thinking Inventory (CTI; Epstein \& Meier, 1989) and the Personal Functioning Inventory (PFI; Kohn et al., 1998). The CTI was designed to assess the extent to which subjects are able to think automatically in ways that help them solve problems of everyday living efficiently and with minimal stress (Epstein \& Meier, 1989). Higher scores on the CTI have been correlated with greater success in social relationships, love, and work, and with greater physical and emotional well-being, characteristics that should also typify the controllability aware. The Personal Functioning Inventory (PFI), a 30-item measure of adaptiveness in coping (Kohn et al., 1998), grew out of Kohn's (1996) view of adaptiveness as involving judging the subjective controllability of situations, then acting with determination in response to what is judged controllable and using self-control to accept what is judged as uncontrollable. Although a preponderance of the items assesses the extent to which the subject is able to manage the emotional aspects of situations that are not controllable, adaptiveness is sufficiently compatible with the ideas underlying controllability awareness that the PFI was a useful guide in developing the CAI.

Although the Constructive Thinking construct does not address controllability explicitly, it encompasses many ideas that closely parallel those underlying Controllability Awareness. Epstein has developed this construct extensively and has carefully fashioned the measurement tool to assess it. The CAI was patterned after the CTI, capitalizing on Epstein's rigorous development process rather than duplicating it. 
The CAI measures the extent to which the individual's behavioral and emotional responses to daily life situations, which may or may not be appraised as stressful, reflect awareness of the controllable and uncontrollable aspects of the outcomes of those situations without focusing explicitly on controllability distinctions. The inventory consists of 20 simple statements, five referring to each of the four aspects of controllability. (1) "Personal control" includes statements relating to the individual's confidence in managing his or her responsibilities. (2) "Shared control" includes statements relating to the individual's ability to engage effectively in interpersonal situations in which the outcome depends on sharing responsibility or control between the individual and another person or persons. (3) "Others in control" includes statements relating to the individual's willingness to accept the uncontrollability of situations that are outside his or her responsibility or authority. (4) "No one in control" includes statements relating to the individual's ability to accept outcomes that are unpredictable or uncontrollable. Half the statements are worded pro-trait; half are worded anti-trait. Subjects indicate how true or false each statement is for them on a five-point scale. Responses to the different aspects of controllability may or may not be correlated depending on the personality characteristics of the individual. Some individuals may be equally adept or inept at accepting the uncontrollable as acting effectively to realize the controllable outcomes, whereas others may be more able in one aspect than another. The four aspects are represented equally in the inventory without the expectation that these groups of items would constitute distinct non-overlapping sub-components of the construct. Thus the CAI is a single inventory without subscales, and no factor analysis was conducted because despite clear differences in the aspects of controllability, we did not expect that responses to these different aspects would emerge as distinct factors. The statements are listed in the Appendix.

The pilot version of the CAI consisted of 24 statements (six from each of the four aspects of controllability) written by one of the authors (J.T.H.). These statements were translated into Hebrew by the other author (E.S.) and verified through a back translation by another translator. An independent judge rated both versions before they were given to subjects. The Hebrew version was then given to 16 unpaid student volunteers, who completed the CAI along with several other inventories at home. Based on the alpha with item deleted for each statement, the items were either reworded or dropped from the inventory, leaving a 20-item list with five items from each of the four controllability awareness categories.

Of the existing assessment tools, the CTI provides a thoroughly reasoned and fully validated measure of ways of thinking that parallels the Controllability Awareness construct, thus it was chosen as the primary comparator in validating the CAI. The PFI was selected as a predictive measure for use in this study because it is a reliable and valid measure of coping ability that also specifically addresses controllability, and therefore can be used to validate the CAI. Thus the CTI and the PFI were used both to guide the development of the CAI and to validate it.

Kobasa's (1979) hardiness scale measures three personality components (labeled "commitment", "challenge", and "control") that together mitigate the effects of stressful life events on health. A slightly modified short form (30 items) of the hardiness scale (Bartone, Ursano, Wright, \& Ingraham, 1989) was used in this study as a predictive measure for comparison with the CAI. The Internal, Powerful Others, and Chance (IPC) scale is a 24-item locus of control scale with three subscales that distinguish between the tendency to attribute causality of events to oneself, to other powerful people, or to chance (Levenson, 1981). The IPC was included to determine whether controllability awareness is a better predictor of adverse outcomes (perceived stress and 
physical symptoms) than locus of control alone. The hardiness scale and the IPC scale were used to compare the predictive value of the CAI with other established measurement tools.

The Perceived Stress Scale (PSS) is a 14-item scale designed to assess subjects' appraisal of how stressful their life situation feels to them (Cohen et al., 1983). The PSS has been recommended for assessing nonspecific appraised stress because it is a better predictor than life event scores. Stresstolerant individuals should have lower perceived stress scores than those lacking stress-tolerance skills. The Cohen-Hoberman Inventory of Physical Symptoms (CHIPS) is a list of 39 physical symptoms selected specifically to exclude psychological symptoms, such as depression and anxiety, but to include complaints of a psychosomatic nature, such as headaches and acid stomach (Cohen \& Hoberman, 1983). It has been shown to have adequate reliability in student populations and is predictive of use of student health services during the 7 weeks following completion of the scale. The PSS and the CHIPS were used as measures of adverse outcomes that are associated with stress intolerance.

\section{Results}

The initial analysis indicated no significant differences between the scores for the men and the women or between the scores or the reliabilities for the English and Hebrew versions of the CAI or the measures of perceived stress and health, thus the results are reported irrespective of the subjects' gender or language. The means and the alpha reliabilities of the predictor measures are summarized in Table 1. The overall reliabilities of 0.78 and 0.85 for the CAI indicate the reliability of this new inventory. The mean scores for the four aspects of controllability are presented for comparison. The scores suggest that subjects rate themselves as slightly better at managing the aspects of situations with some degree of control than those aspects that are uncontrollable.

The correlations between the CAI, the PFI, and the CTI with the measures of perceived stress and physical symptoms for the 42 subjects who completed these five measures are presented in Table 2. Although all the correlations were significant, the overall score for the CAI was slightly more highly correlated with the PSS and the CHIPS than the scores on either the PFI or the CTI. The high correlations among the CAI, the PFI, and the CTI indicate the similarities of these measures and validate the CAI as an effective measure of adaptive responses, yet the multiple regression (see later) distinguishes among them. The mean score on the PFI (102.4) and the alpha reliability (0.90) were comparable to the means (101.59 and 101.86) and alpha reliabilities $(0.92$ and 0.89 ) reported by Kohn et al. (1998). The mean score on the CTI (57.21) and the alpha reliability $(0.83)$ were comparable to the norms (mean $=58.47$; alpha reliability $=0.86)$ established by Epstein (personal communication) for the short form of the CTI. This suggests that the interpretations of the Hebrew translations of the PFI and the CTI by Israeli subjects were comparable to the interpretations of the original English inventories by English speakers.

The correlations of the CAI-retest, the PFI, the CTI, the IPC, the hardiness scale, and the hardiness sub scales with the measures of perceived stress and physical symptoms for the 25 subjects who completed the retest are summarized in Table 3. Again the CAI had the highest correlation with both the PSS and the CHIPS. Although the correlation between the hardiness scale and the PSS matched that of the CAI, the hardiness scale, contrary to expectation, was not significantly correlated with the CHIPS. The correlation results suggest that the CAI is a better 
predictor of both adverse outcomes (perceived stress and physical symptoms) than the other measures tested.

Because the inventories selected for comparison with the new CAI in this study were all proven measures, it was important to determine whether the CAI makes a unique contribution to predicting perceived stress and physical symptoms. A series of stepwise multiple regressions (for the CAI, PFI, and CTI in the first test and for the CAI, PFI, CTI, and hardiness scale in the retest) was conducted to determine the relative contribution of each inventory to predicting adverse

Table 1

Descriptive statistics and alpha reliabilities for the predictive measures ${ }^{\mathrm{a}}$

\begin{tabular}{|c|c|c|c|c|}
\hline & Valid $N$ & Mean & S.D. & $\alpha$ Reliability \\
\hline $\mathrm{CaI}$ & $42^{\mathrm{b}}$ & 63.83 & 9.47 & 0.78 \\
\hline $\mathrm{CAI}$ - personal control & 42 & 17.14 & 3.84 & \\
\hline CAI-shared control & 42 & 16.74 & 2.71 & \\
\hline $\mathrm{CAI}$ - others in control & 42 & 14.29 & 2.74 & \\
\hline CAI - uncontrollable & 42 & 15.67 & 3.43 & \\
\hline $\mathrm{CAI}$-retest & $25^{\mathrm{c}}$ & 64.08 & 10.14 & 0.85 \\
\hline CAIR - personal control & 25 & 16.68 & 3.00 & \\
\hline CAIR — shared control & 25 & 16.76 & 2.47 & \\
\hline CAIR - others in control & 25 & 15.04 & 3.16 & \\
\hline CAIR - uncontrollable & 25 & 15.60 & 3.92 & \\
\hline PFI & 42 & 102.40 & 17.47 & 0.90 \\
\hline CTI & 42 & 57.21 & 10.91 & 0.83 \\
\hline IPC-Internal & 25 & 35.92 & 4.89 & 0.67 \\
\hline IPC-Powerful others & 25 & 16.28 & 5.92 & 0.61 \\
\hline IPC-Chance & 25 & 21.40 & 7.95 & 0.78 \\
\hline Hardiness & 25 & 58.12 & 6.48 & 0.69 \\
\hline Hardy-Commitment & 25 & 20.64 & 3.04 & 0.55 \\
\hline Hardy_Challenge & 25 & 18.16 & 2.95 & 0.49 \\
\hline Hardy-Control & 25 & 19.32 & 2.85 & 0.43 \\
\hline
\end{tabular}

a CAI, Controllability Awareness Inventory; CAIR, Controllability Awareness Inventory-retest; PFI, Personal Functioning Inventory; CTI, Constructive Thinking Inventory; IPC, Internal, Powerful Others, and Chance scale (with subscales); Hardiness, the hardiness scale; Hardy, subscales of the hardiness scale.

b $N=8$ English and 34 Hebrew.

c $N=3$ English and 22 Hebrew.

Table 2

Correlations among the CAI, the PFI, the CTI, the PSS, and the CHIPS

\begin{tabular}{llllll}
\hline & PSS & CHIPS & CAI & PFI & $0.66^{* *}$ \\
\hline CaI & $-0.60^{* *}$ & $-0.52^{*}$ & - & - & $0.63^{* *}$ \\
PFI & $-0.59^{* *}$ & $-0.46^{*}$ & $0.66^{* *}$ & $0.80^{* *}$ & $0.80^{* *}$ \\
CTI & $-0.47^{*}$ & $-0.43^{*}$ & $0.63^{* *}$ & - &
\end{tabular}

a PSS, Perceived Stress Scale; CHIPS, Cohen-Hoberman Inventory of Physical Symptoms; CaI, Controllability Awareness Inventory; PFI, Personal Functioning Inventory; CTI, Constructive Thinking Inventory

$* P<0.01$.

** $P<0.001$ 
outcomes. The CAI was the only variable that remained in the equation as significant for both test groups for both the PSS $\left(R^{2}=0.36, F(1,40)=22.31, P<0.0001 ; R^{2}=0.62, F(2,22)=17.94\right.$, $P<0.0001)$ and the CHIPS $\left(R^{2}=0.27, F(1,40)=14.97, P<0.0004 ; R^{2}=0.48, F(1,23)=21.45\right.$, $P<0.0001)$. The hardiness scale also remained significant as a comparable predictor of perceived stress, but in this study it was not a significant predictor of physical symptoms.

The correlation between the scores on the test-retest of the CAI for the 25 subjects who completed the inventory twice was $0.76(P<0.0001)$, suggesting that the scores on the CAI are relatively stable over a week. Although the sample sizes in the initial groups were small, these results are sufficiently robust to demonstrate the reliability, validity, and predictive value of the CAI.

An initial analysis revealed some differences in the scores for the men and women in both the US and the NZ samples: the men's scores were higher than the women's on the CAI (US: 66.63 vs. 62.48 ; NZ: 71.39 vs. 66.57 ) and were lower on the PSS (US: 23.61 vs. 27.76; NZ: 21.03 vs. 25.22) and the CHIPS (US: 20.18 vs. 31.70 ; NZ: 20.24 vs. 28.92 ). The results are presented together, however, because there were no differences in the correlations when calculated separately by gender and because the purpose of this study was to investigate the relationships between controllability awareness and adverse outcomes rather than to assess gender differences in any of the measures. The initial analysis also indicated significant differences between the average CAI scores for the US and the NZ samples. Although there were no substantial differences in the correlations between the CAI and the PSS or the CHIPS, the results are reported separately for the two subject groups. The alpha reliabilities for the CAI of 0.84 in both the US and the NZ samples confirm the reliability of this new inventory. The correlations of the CAI with the measures of perceived stress and physical symptoms were significant at $P<0.001$ in both samples (CAI with PSS: US:-0.68; NZ:-0.68; CAI with CHIPS: US:-0.46; NZ:-0.50). The correlations calculated separately for the four aspects of controllability with the PSS ranged from-0.41 to -0.71 and with the CHIPS ranged from -0.26 to -0.46 ; all were significant at $P<0.001$.

Table 3

Correlations of the CAI-retest and other predictive measures with the PSS and CHIPS ${ }^{\text {a }}$

\begin{tabular}{lll}
\hline & PSS & CHIPS \\
\hline CAI-retest & $-0.65^{* * *}$ & $-0.69^{* * *}$ \\
PFI & $-0.63^{* * *}$ & $-0.52^{* *}$ \\
CII & $-0.63^{* * *}$ & -0.42 \\
IPC-Internal & -0.22 & -0.25 \\
IPC-Powerful others & -0.35 & -0.31 \\
IPC-Chance & $-0.40^{*}$ & -0.27 \\
Hardiness & $-0.65^{* * *}$ & -0.25 \\
Hardy_Commitment & $-0.53^{* *}$ & -0.15 \\
Hardy_Challenge & $-0.50^{*}$ & -0.12 \\
Hardy_Control & $-0.40^{*}$ & -0.29
\end{tabular}

a PSS, Perceived Stress Scale; CHIPS, Cohen-Hoberman Inventory of Physical Symptons; CAI, Controllability Awareness Inventory; PFI, Personal Functioning Inventory; CTI, Constructive Thinking Inventory; IPC, Internal, Powerful Others, and Chance scale (with subscales); Hardiness, the hardiness scale; Hardy, subscales of the hardiness scale.

* $P<0.05$.

** $P<0.01$.

*** $P<0.001$. 


\section{Discussion}

The results support the hypothesis that controllability awareness is associated with stress tolerance by demonstrating a clear relationship between using controllability awareness in response to life situations and lower levels of adverse outcomes (perceived stress and physical symptoms) in students from three continents. Significant correlations between each of the four aspects of controllability and the measures of adverse outcomes indicate that each aspect contributes to predicting stress tolerance as it is manifest in lower perceived stress and fewer physical symptoms. Assessing controllability awareness as a combination of responses to these four aspects of controllability enables clear predictions of adverse outcomes even with a short inventory.

The traditional locus of control construct (Levenson, 1981; Rotter, 1966) places the controllable and the uncontrollable at separate ends of a spectrum thus it may seem odd to measure controllability as a composite of controllable and uncontrollable aspects. However, the results from the CAI indicate that assessing responses in terms of a combination of aspects, two (personal control and shared control) having to do with effective action (yet distinguishing between action when the individual is personally in control and when the control of the outcome is shared with one or more other individuals) and two (others in control and uncontrollable) having to do with effective acceptance of uncontrollability (yet distinguishing between acceptance of others being in control and acceptance of no one being in control) is a more effective predictor of adverse outcomes than the Internal, Powerful Others, and Chance scale (Levenson, 1981). The distinction between others in control as others being "responsible" rather than others being "powerful" adds subtlety to the assessment of accepting situations when one is not personally in control of the outcome.

The concept of shared control in social interactions when the outcome depends on mutual responsibility of two or more individuals may be an important addition to the controllability construct. After all, humans are social beings who interact almost constantly with one another. Most sources of both distress and comfort come from relationships, thus it is prudent to include this aspect of controllability in constructs designed to characterize stress tolerance.

In their pioneering studies of the importance of cognitive appraisal on the emotional and physiological responses to environmental demands, Lazarus and his colleagues (see Lazarus, 1991, for a review) distinguished between stress responses (high negative affect and sympathetic nervous system activation) to threat appraisals, when the environmental demands were assessed as exceeding the individual's coping resources, and effective responses (positive affect and effective mobilization of energy resources) to challenge appraisals, when the environmental demands were assessed as within the individual's ability to cope. The theoretical basis of controllability awareness as a characteristic of stress tolerance is rooted in the same distinction. Individuals who are either unaware of or are unable to distinguish between those aspects of situations that are controllable and those that are not would be more likely to appraise environmental demands as threatening. In contrast, individuals who are aware of these distinctions are more likely to appraise environmental demands as challenges because their controllability analysis immediately categorizes the situation based on achievable and futile outcomes. Identifying the areas with achievable outcomes enhances motivation to undertake those challenges whereas failure to make such distinctions leaves the situation as an unanalyzed threat. Controllability aware individuals evaluate the challenge and determine what the most effective response will be; individuals who are 
not aware of the controllability may not be able to get beyond the threat. It follows that controllability awareness would be associated with lower perceived stress (i.e. fewer threat appraisals) because of the tendency to act effectively based on the controllability analysis and to accept what cannot be controlled. Research on the relationship between stress responses and compromised effectiveness of the immune system (Kiecolt-Glaser \& Glaser, 1994) suggests that continued threat appraisals have deleterious effects on health, thus individuals who are not aware of the controllability of outcomes may be more likely to suffer from physical symptoms than those who make controllability distinctions and thereby experience less stress. The results are compatible with the broad hypothesis motivating this research.

If the relationship between greater controllability awareness and less perceived stress and fewer physical symptoms is causal as well as correlational, it follows that training in controllability awareness could enable individuals who have been experiencing life stress and stress-related symptoms to reduce their perceived stress and the associated physical symptoms by helping them to identify environmental demands as challenges rather than threats.

It will be important to corroborate the evidence presented here with results from additional research testing the CAI in samples from different populations, including working adults, patients suffering from chronic and acute physical and psychological illnesses, and cross-cultural groups with varying types of environmental stressors (e.g. Somer, Keinan, \& Carmil, 1996). It will also be important to explore the relationships between controllability awareness and personality variables to broaden the theoretical basis of the construct. Investigations of the physiological correlates of varying degrees of controllability awareness will also be needed to validate these hypotheses beyond the results from self-report measures. Results from these additional studies will deepen the understanding of controllability awareness and its contribution to stress tolerance under different environmental circumstances. Ongoing research is addressing these important lingering questions.

\section{Acknowledgements}

We are grateful to S. Breznitz for fruitful discussions in the early stages of this research and to S. Epstein, C. Gordon, and P. Kohn for helpful comments on an earlier version of the manuscript. We thank G. Heth and T. Myers for assistance in rating response effectiveness and $\mathrm{T}$. Mencher and G. Heth for assistance in translation and back-translation. We thank M. Block, D. Barkmeier, K. Spikes, N. Blampied, M. Barnes, and S. Katzir for assistance in recruiting subjects. This research was supported in part by a matching grant from the Israel Ministry of Absorption and the Research Authority of the University of Haifa to J. Todrank Heth.

\section{Appendix}

\section{Personal control:}

3. I have the determination necessary to succeed. $(+)$

5. I am confident that I can manage all the things I have to do. $(+)$ 
7. I worry that I won't be able to finish all the things I need to do. (-)

15. I don't get discouraged when I have difficult problems to solve. (+)

20. Sometimes my responsibilities are too much for me. (-)

\section{Shared control:}

4. I get angry when I can't convince someone that I am right. (-)

6. I try to stay objective during interpersonal discussions. $(+)$

9. I can't keep calm when a friend has a different opinion. (-)

13. I get upset when someone won't cooperate with me. (-)

19. I usually don't let criticism bother me too much. $(+)$

\section{Others in control:}

1. I can't relax when I'm waiting to hear about an important decision. (-)

8. I get upset by the slightest sign of disapproval by others. (-)

11. I can let others make their own decisions without interfering. $(+)$

16. I try not to be impatient with inefficient clerks and administrators. $(+)$

18. I lose my temper when I think other people may be trying to take advantage of me. (-)

\section{Uncontrollable:}

2. I don't worry about things if there's nothing I can do about them. $(+)$

10. I can keep my mind off bad things that might happen. $(+)$

12. I try not to worry when there is no way to change how things will turn out. $(+)$

14. When I'm unlucky, it bothers me for a long time. (-)

17. I can't think about other things while waiting to find out about something important. (-)

\section{References}

Antonovsky, A. (1987). Unraveling the mystery of health: how people manage stress and stay well. San Francisco: Jossey-Bass.

Bandura, A. (1982). Self-efficacy mechanism in human agency. American Psychologist, 37(2), $122-147$.

Bartone, P. T., Ursano, R. J., Wright, K. M., \& Ingraham, L. H. (1989). The impact of a military air disaster on the health of assistance workers. The Journal of Nervous and Mental Disease, 177(6), 317-328.

Carver, C. S., \& Scheier, M. F. (1994). Situational coping and coping dispositions in a stressful transaction. Journal of Personality and Social Psychology, 66, 184-195.

Carver, C. S., Scheier, M. F., \& Weintraub, J. K. (1989). Assessing coping strategies: a theoretically based approach. Journal of Personality and Social Psychology, 56, 267-283.

Cohen, S., \& Herbert, T. B. (1996). Health psychology: psychological factors and physical disease from the perspective of human psychoneuroimmunology. Annual Review of Psychology, 47, 113-142.

Cohen, S., \& Hoberman, H. (1983). Positive events and social support as buffers of life change stress. Journal Applied Social Psychology, 13, 99-125. 
Cohen, S., Kamarck, T., \& Mermelstein, R. (1983). A global measure of perceived stress. Journal of Health and Social Behavior, 24(3), 85-396.

Epstein, S., \& Katz, L. (1992). Coping ability, stress, productive load, and symptoms. Journal of Personality and Social Psychology, 62(5), 813-825.

Epstein, S., \& Meier, P. (1989). Constructive thinking: a broad coping variable with specific components. Journal of Personality and Social Psychology, 57(2), 332-350.

Folkman, S. (1984). Personal control and stress and coping processes: a theoretical analysis. Journal of Personality and Social Psychology, 46(4), 839-852.

Kiecolt-Glaser, J. K., \& Glaser, R. (1994). Handbook of human stress and immunity. San Diego: Academic Press.

Kobasa, S. C. (1979). Stressful life events, personality, and health: an inquiry into hardiness. Journal of Personality and Social Psychology, 37(1), 1-11.

Kohn, P. M. (1996). On coping adaptively with daily hassles. In M. Zeidner, \& N. S. Endler (Eds.), Handbook of coping: theory, research, application. New York: Wiley.

Kohn, P. M., O'Brien-Wood, C., Pickering, D. I., \& De Cicco, T. L. (1998). The Personal Functioning Inventory: A reliable and valid measure of adaptiveness in coping. Unpublished manuscript, York University, Toronto.

Lazarus, R. S. (1991). Emotion and adaptation. New York: Free Press.

Lazarus, R. S., \& Folkman, S. (1984). Stress, appraisal, and coping. New York: Springer.

Levenson, H. (1981). Differentiating among internality, powerful others, and chance. In H. M. Lefcourt (Ed.), Research with the locus of control construct: Assessment methods. New York: Academic Press.

Miller, S. M. (1992). Individual differences in the coping process: what to know and when to know it. In B. N. Carpenter (Ed.), Personal coping: theory, research, and application. Westport, CN: Praeger.

Peterson, C., \& Seligman, M. E. P. (1987). Explanatory style and illness. Journal of Personality and Social Psychology, $55,237-265$.

Peterson, C., \& Stunkard, A. J. (1989). Personal control and health promotion. Social Scientific Medicine, 28(8), 819828.

Rotter, J. (1966). Generalized expectancies for internal versus external control of reinforcement. Psychological Monographs, 80, 609.

Scheier, M. F., \& Carver, C. S. (1992). Effects of optimism on psychological and physical well-being: theoretical overview and empirical update. Cognitive Therapy and Research, 16(2), 201-228.

Scheier, M. F., Weintraub, J. K., \& Carver, C. S. (1986). Coping with stress: divergent strategies of optimists and pessimists. Journal of Personality and Social Psychology, 51, 1257-1264.

Skinner, E. (1996). A guide to constructs of control. Journal of Personality and Social Psychology, 71(3), 549-570.

Somer, E., Keinan, G., \& Carmil, D. (1996). Psychological adaptation of anxiety disorder patients following repeated exposure to emergency situations. Journal of Traumatic Stress, 9(2), 207-221.

Thompson, S. C., \& Spacapan, S. (1991). Perceptions of control in vulnerable populations. Journal of Social Issues, $47(4), 1-21$. 